\title{
Erratum
}

\section{The fundamental plane of radio galaxies ${ }^{\star}$}

\author{
D. Bettoni ${ }^{1}$, R. Falomo ${ }^{1}$, G. Fasano ${ }^{1}$, F. Govoni ${ }^{2}$, M. Salvo ${ }^{1}$, and R. Scarpa ${ }^{3}$ \\ 1 Osservatorio Astronomico di Padova, Vicolo Osservatorio 5, 35122 Padova, Italy \\ e-mail: bettoni@pd.astro.it; falomo@pd.astro.it; fasano@pd.astro.it; salvo@pd.astro.it \\ 2 Istituto di Radioastronomia di Bologna and Dipartimento di Astronomia, Universitá di Bologna, Italy \\ e-mail: fgovoni@ira.bo.cnr.it \\ ${ }^{3}$ European Southern Observatory \\ e-mail: rscarpa@eso.org
}

A\&A, 380, 471-477 (2001), DOI: 10.1051/0004-6361:20011486

In this paper we found the following errors introduced in proof:

- In Table 3 Cols. 3 and 4 report incorrect data for the Smith et al. (1990) and Faber et al. (1989) sample; in the following Table 1 we report the correct values;

- In Fig. 2 the label of the $Y$-axis is: $1.242 \log \sigma+0.33<\mu_{\mathrm{e}}>-8.581$.
Send offprint requests to: D. Bettoni, e-mail: bettoni@pd . astro. it

$\star$ Based on observations collected at European Southern Observatory, La Silla, Chile.
Table 1. Radio galaxies from literature.

\begin{tabular}{|c|c|c|c|c|}
\hline name & $\begin{array}{l}\log \sigma \\
\mathrm{km} \mathrm{s}^{-1}\end{array}$ & $\begin{array}{c}\log r_{\mathrm{e}} \\
\mathrm{kpc}\end{array}$ & $<\mu_{\mathrm{e}}>$ & $\begin{array}{c}V_{\mathrm{r}} \\
\mathrm{km} \mathrm{s}^{-1}\end{array}$ \\
\hline \multicolumn{5}{|c|}{ Smith et al. (1990) } \\
\hline 3 C 29 & 2.318 & 1.180 & 20.29 & 13400 \\
\hline $3 C 31$ & 2.394 & 1.183 & 20.79 & 5007 \\
\hline $3 C 33$ & 2.362 & 1.066 & 20.43 & 17840 \\
\hline $3 \mathrm{C} 62$ & 2.436 & 1.264 & 21.07 & 44370 \\
\hline 3C 76.1 & 2.301 & 0.784 & 19.28 & 9713 \\
\hline 3C 78 & 2.417 & 1.094 & 19.44 & 8634 \\
\hline $3 C 84$ & 2.391 & 1.234 & 20.37 & 5156 \\
\hline $3 \mathrm{C} 88$ & 2.276 & 1.370 & 21.93 & 9054 \\
\hline $3 C 89$ & 2.398 & 1.610 & 22.51 & 41550 \\
\hline 3C 98 & 2.238 & 1.020 & 20.84 & 9174 \\
\hline $3 \mathrm{C} 120$ & 2.301 & 1.146 & 20.51 & 10010 \\
\hline 3C 192 & 2.283 & 0.940 & 20.06 & 17930 \\
\hline 3С 196 & 2.322 & 1.464 & 21.23 & 59360 \\
\hline $3 C 223$ & 2.305 & 1.192 & 21.03 & 41010 \\
\hline 3C 293 & 2.267 & 0.876 & 18.99 & 13550 \\
\hline 3C 305 & 2.250 & 0.744 & 18.13 & 12470 \\
\hline 3C 338 & 2.462 & 1.562 & 21.48 & 9084 \\
\hline 3C 388 & 2.562 & 1.643 & 21.86 & 27220 \\
\hline $3 \mathrm{C} 444$ & 2.190 & 1.763 & 22.62 & 45870 \\
\hline 3C 449 & 2.350 & 1.423 & 22.83 & 5126 \\
\hline PKS 0634-206 & 2.290 & 0.878 & 18.70 & 16790 \\
\hline PKS 2322-122 & 2.350 & 1.477 & 21.62 & 24610 \\
\hline \multicolumn{5}{|c|}{ Faber et al. (1989) } \\
\hline NGC 315 & 2.493 & 1.414 & 20.58 & 5126 \\
\hline NGC 741 & 2.447 & 1.415 & 20.99 & 5546 \\
\hline NGC 4839 & 2.387 & 1.311 & 21.41 & 6955 \\
\hline NGC 7626 & 2.511 & 1.044 & 20.85 & 7615 \\
\hline $3 \mathrm{C} 40$ & 2.233 & 0.640 & 18.18 & 5426 \\
\hline
\end{tabular}

